\title{
Maturidade de potros nascidos de éguas com placentite
}

\author{
[Maturity of foals from the mares with placentitis] \\ L.S. Feijó, B.R. Curcio, C. Haetinger, F.M. Pazinato, S. Kasinger, R.S. Dos Santos, \\ S.R.L. Ladeira, C.E.W. Nogueira \\ Universidade Federal de Pelotas - Campus Capão do Leão - Pelotas, RS \\ RESUMO
}

\begin{abstract}
Objetivou-se descrever a maturidade neonatal através da resposta clínica, comportamental e hematológica de potros nascidos de éguas com placentite. Participaram do estudo seis potros nascidos de éguas submetidas à indução experimental de placentite ascendente através da infusão intracervical de Streptococcus equi subespécie zooepidemicus e tratadas com Sulfa-trimetoprim e Flunixin meglumine. A formação dos grupos neonatais foi realizada de acordo com o grau de viabilidade e sobrevivência até 60 horas: Grupo Não Sobreviventes $(n=2)$; Grupo Debilitados $(n=2)$; Grupo Saudáveis $(n=2)$. Foi considerado o tempo de gestação, período de intervalo inoculação-parto, avaliação comportamental,

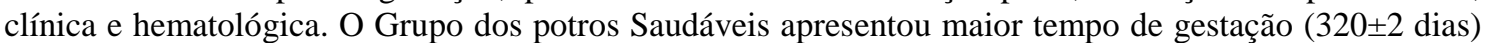
e maior intervalo inoculação-parto (20,5 $\pm 2,5$ dias). Os Grupos Não Sobreviventes e Debilitados apresentaram atraso para decúbito esternal e reflexo de sucção. Foi observada bradicardia e hipotermia com 48h de vida no Grupo Não Sobreviventes. Os potros do Grupo Não Sobreviventes e Saudáveis apresentaram leucopenia no nascimento com discretas variações até as $48 \mathrm{~h}$. Os potros nascidos de éguas com placentite ascendente e tratadas demonstraram evolução clínica e respostas neonatais distintas. Conclui-se que, quanto maior o tempo de manutenção da gestação após a injúria placentária, melhor será a maturação fetal, o que refletirá em viabilidade e melhor capacidade de resposta neonatal.
\end{abstract}

Palavras-chave: equinos, gestação, neonatos, imaturidade, hematologia

\begin{abstract}
The aim of this study was to describe the neonatal maturity through clinical, behavioral and hematologic response of foals born from mares with placentitis. Were used six foals born from mares subjected to experimentally induced ascending placentitis through intracervical infusion of Streptococcus equi subspecies zooepidemicus and treated with trimethoprim sulfametoxazole and flunixin meglumine. The neonatal groups were performed according to the viability and survival rate up to 60 hours: No Survivors group $(n=2)$; Debilitated group $(n=2)$; Healthy group $(n=2)$. Gestational length, the period between inoculation and delivery, and behavioral, clinical and hematologic evaluations were considered. The

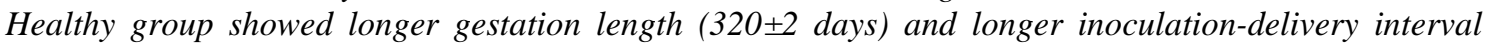
(20.5 \pm 2.5 days). No Survivors and Debilitated groups showed delay in sternal recumbency and sucking reflex. Bradycardia and hypothermia was observed at 48 hours of life in No Survivors Group. Foals from No Survivors and Healthy groups showed leukopenia at birth with slight variations until 48h. Foals born from mares with ascending placentitis and treated showed distinct clinical and neonatal responses. It is concluded that the longer the maintenance of gestation after placental injury, better is the fetal maturation, which reflects in better viability and ability to neonatal response.
\end{abstract}

Keywords: equine, pregnancy, neonatal, immaturity, hematology

Recebido em 6 de maio de 2014

Aceito em 12 de novembro de 2014

E-mail: feijo.lorena.s@gmail.com 


\section{INTRODUÇÃo}

A prontidão para o nascimento ocorre com a ação do eixo hipotalâmico-hipofisiarioadrenal fetal, que, através da produção de cortisol, atua na maturação do sistema endócrino e demais sistemas orgânicos. Esse processo ocorre durante as últimas 48-72 horas pré-parto (Rossdale e Silver, 1982). Sem a maturação do córtex adrenal, o potro torna-se incapaz de sobreviver no ambiente pós-natal, ainda que seja realizado tratamento de suporte necessário (Rossdale et al., 1997). Estudos clínicos demonstram que a maturação fetal pode ser iniciada de forma precoce, em situações nas quais o feto é submetido a estresse crônico, como o desenvolvimento de lesões placentárias e episódios de hipóxia (Rossdale et al., 1991; Mays et al., 2002).

A causa mais frequente de partos prematuros e morte neonatal em equinos está associada à placentite. $\mathrm{O}$ comprometimento na unidade uterofetoplacentária, por processos de hipoxemia ou infecção, reduz o aporte de nutrientes e oxigênio para o feto e placenta (Giles et al., 1993; Hong et al., 1993). Essas alterações podem causar danos na maturação e limitação do desenvolvimento fetal, reduzindo as chances do nascimento de um potro viável. No entanto, potros nascidos de éguas com comprometimento placentário podem ser prematuros, apresentar alteração clínica incompatível com a vida ou, ainda, podem nascer a termo e com pouca ou nenhuma alteração (Bain, 2004).

Objetivou-se descrever a maturidade neonatal através da resposta clínica, comportamental e hematológica de potros nascidos de éguas com placentite.

\section{MATERIAL E MÉTODOS}

O estudo foi realizado com o plantel de éguas do Centro de Ensino e Experimentação em Equinocultura da Palma (CEEP), da Universidade Federal de Pelotas, no município de Capão do Leão, Rio Grande do Sul (314'08.2"S; 52²9'51.4"O), durante a temporada reprodutiva 2012-2013. Para o estudo foram utilizadas seis éguas mestiças Crioulas com idade média $11 \pm 2$ anos e média de peso $437 \pm 22 \mathrm{~kg}$.
As éguas eram submetidas a exame clínico geral, acompanhamento hematológico e exame obstétrico completo, no qual eram avaliadas as membranas placentárias, desenvolvimento e atividade fetal. As gestações selecionadas para esse estudo não apresentaram alterações clínicas e nos exames complementares até o momento de indução da placentite ascendente.

A indução de placentite foi realizada através da infusão intracervical de Streptococcus equi subespécie zooepidemicus na concentração de $10^{7}$ UFC, entre os dias 280-295 de gestação, conforme protocolo descrito por Bailey et al. (2010). Após a indução, as éguas eram submetidas a monitoramento intensivo até 0 momento do parto.

Todas as éguas apresentaram sinais clínicos de placentite, como secreção vulvar purulenta a sanguinolenta, além de desenvolvimento precoce do úbere. Foi observado espessamento da junção uteroplacentária (JUP) e descolamento placentário 24-48h após a inoculação bacteriana. Nesse mesmo período pós-indução foi identificado crescimento de $S$. equi zooepidemicus no exame microbiológico e presença de células inflamatórias na avaliação citológica, confirmando o desenvolvimento clínico de placentite ascendente.

O protocolo de tratamento era iniciado 48h após a indução de placentite e consistia na administração de Sulfa-trimetoprim (Trissulfin ${ }^{\circledR}$, sulfametoxazol e trimetoprim, Ouro Fino Agronegócio, Brasil), na dose $30 \mathrm{mg} / \mathrm{kg}$, a cada 12 horas, durante 10 dias e Flunixin meglumine (Desflan $^{\circledR}$, Flunixin meglumine, Ouro Fino Agronegócio, Brasil), na dose $1.1 \mathrm{mg} / \mathrm{kg}$, a cada 24 horas, com duração de sete dias.

Todos os partos foram assistidos. Não foi observado nenhum caso de distocia; porém, todas as éguas apresentaram descolamento precoce da placenta, em que foi procedida intervenção com rompimento da membrana corioalantoide e auxílio na tração do potro. Imediatamente após o nascimento foi realizada avaliação e atendimento ao neonato.

O diagnóstico pós-parto de placentite ascendente foi confirmado na avaliação histopatológica placentária ao identificar focos de inflamação supurativa no corioalantoide e vilosidades nas 
regiões de estrela cervical e corpo uterino, presença difusa de neutrófilos, piócitos e macrófagos dispostos no corioalantoide. Dessa forma, o diagnóstico de placentite ascendente foi confirmado através da observação dos sinais clínicos, ultrassonografia, microbiologia, citologia e, por fim, histopatologia placentária.

Para avaliação da maturidade neonatal no presente estudo, foi considerado o tempo de gestação e o intervalo inoculação-parto (período de manutenção da gestação após a indução de placentite). Na avaliação do potro foi realizada inspeção para observação das características físicas e avaliação dos reflexos posturais e comportamentais, sendo considerado tempo para permanecer em decúbito esternal, apresentação do reflexo de sucção e tempo para se levantar.

Na avaliação clínica foi aferida frequência cardíaca, respiratória e temperatura retal. Ainda foram avaliadas mudanças de comportamento e atitude, viabilidade neonatal e demais alterações decorrentes. Para descrição da apresentação clínica, foi estipulado o momento imediato ao nascimento, 06h, 12h, 24h, 36h e 48h de vida.

A avaliação hematológica foi realizada considerando valores de contagem de hemácias, concentração de hemoglobina, hematócrito, volume corpuscular médio (VCM), contagem de plaquetas, proteínas plasmáticas totais (PPT), fibrinogênio, contagem de leucócitos totais, neutrófilos, linfócitos e taxa neutrófilo:linfócito $(\mathrm{N}: \mathrm{L})$. As coletas de sangue foram feitas através de venopunção jugular em tubos com EDTA, imediatamente após o nascimento, 12h, 24h e $48 \mathrm{~h}$ de vida.

Os neonatos foram tratados com ampicilina (Cilinon ${ }^{\circledR}$, Ampicilina sódica, Ariston, Brasil), na dose $22 \mathrm{mg} / \mathrm{kg}$, a cada 6 horas, flunixin meglumine (Desflan ${ }^{\circledR}$, Flunixin meglumine, Ouro Fino Agronegócio, Brasil), na dose $1.1 \mathrm{mg} / \mathrm{kg}$, a cada 8 horas, e fluidoterapia parenteral aquecida $\left(38^{\circ} \mathrm{C}\right)$ com ringer lactato e glicose 5\% (Fresenius Kabi Brasil Ltda, Brasil), na dose $20 \mathrm{~mL} / \mathrm{kg}$. Nos potros que apresentaram ausência de sucção, foi mantida alimentação enteral, através de sondagem nasogástrica. O colostro foi administrado 60-90 minutos após o nascimento e, posteriormente, leite materno durante todo o período necessário. Potros debilitados e hipotérmicos foram mantidos com cobertores e colchão térmico.

A divisão dos potros foi realizada de acordo com o grau de viabilidade e sobrevivência até 60 horas: Grupo Não sobreviventes $(n=2)$, composto por potros severamente debilitados e não sobreviventes até 60 horas de vida; Grupo Debilitados $(n=2)$, composto por potros doentes, porém viáveis; Grupo Saudáveis $(n=2)$, composto por potros imaturos, porém com resposta satisfatória.

Os dados foram analisados por estatística descritiva através do programa Statistix 8. Todos os dados estão expressos como média \pm erro padrão da média (EPM).

Este estudo foi aprovado pelo Comitê de Ética e Experimentação Animal da Faculdade de Veterinária da UFPel, sob o nº 3891.

\section{RESULTADOS}

A descrição do tempo para apresentação dos reflexos posturais e comportamentais, evolução clínica e resultados neonatais dos grupos estudados estão expressos na Figura 1. Na avaliação do tempo de gestação, foram obtidas as médias de 312 \pm 2 dias para o Grupo Não Sobreviventes; 315 \pm 1 para o Grupo Debilitados;

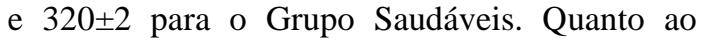
tempo de inoculação-parto, foram obtidas as médias de $12 \pm 2,15 \pm 1$ e $20,5 \pm 2,5$ para os mesmos grupos, respectivamente.

Todos os potros apresentaram padrão físico indicativo de imaturidade, em que foram observadas uma ou mais características, como proeminência frontal, presença de "hoof pads", fraco tônus muscular, flacidez de orelhas e lábios, além de pelos finos e curtos, conforme descrito por Koterba (1990a) e Lester (2005). Na avaliação dos índices médios da relação peso potro / peso égua, foram encontrados: $7,1 \%$ no Grupo Não Sobreviventes; 6,6\% no Grupo Debilitados; e 6,3\% no Grupo Saudáveis. Os valores em quilogramas estão descritos na Figura 1 , assim como os tempos dos reflexos posturais e comportamentais, evolução clínica e resultado neonatal.. 


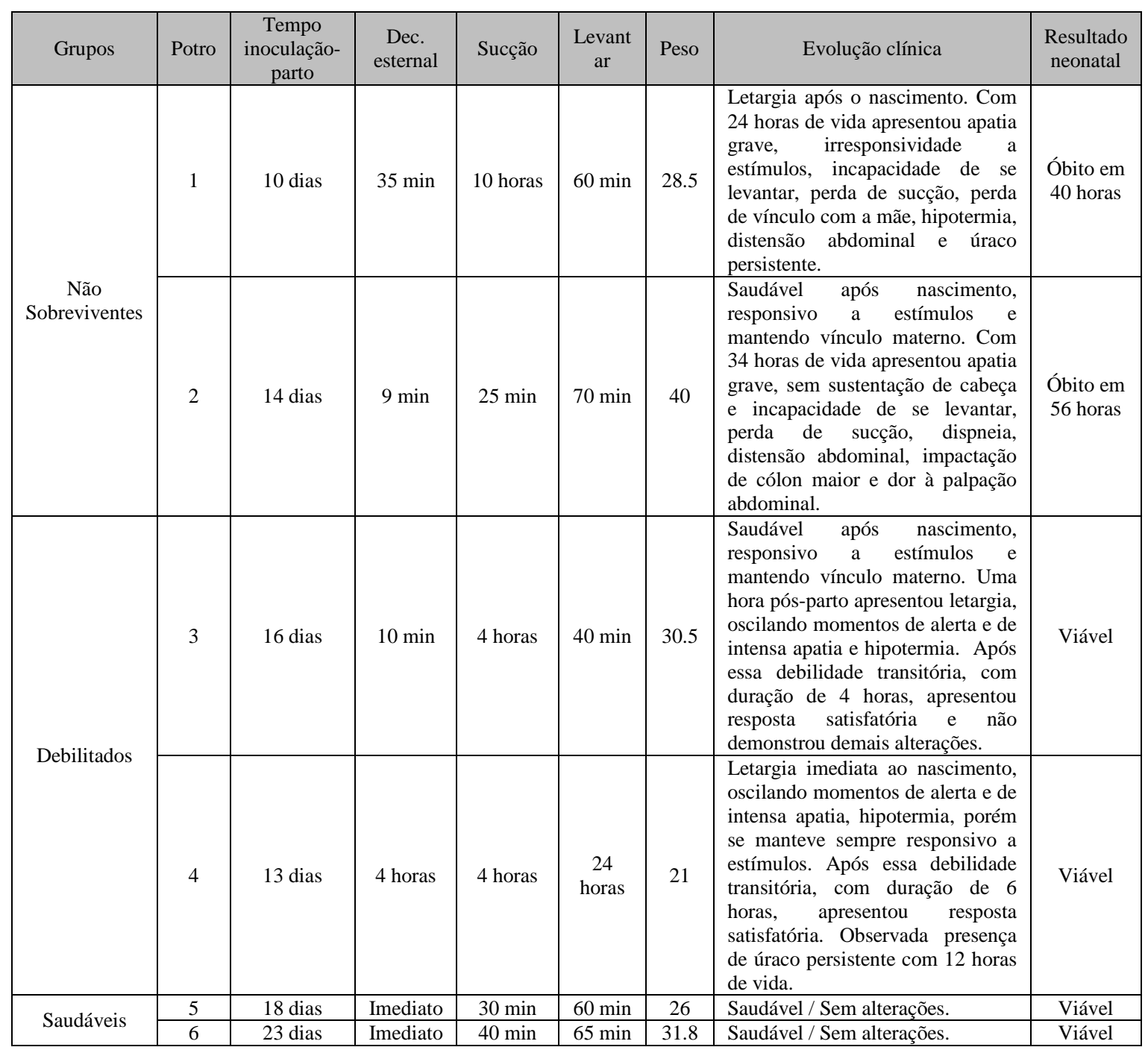

Figura 1. Descrição dos reflexos posturais e comportamentais, evolução clínica e resultados neonatais observados nos potros dos grupos estudados. Peso em quilogramas (kg).

Na apresentação do decúbito esternal, reflexo de sucção e tempo para se levantar, é evidente a imaturidade de alguns potros estudados. Em relação ao quarto neonato, o qual demorou 24 horas para se levantar, foi observada hiperextensão grave nas articulações metacarpo e metatarsofalangeanas, sendo esse atraso decorrente da imaturidade do sistema musculoesquelético.

Ao avaliar a evolução clínica e resultado neonatal, observou-se que os potros do Grupo Não Sobreviventes apresentaram perda dos reflexos adaptativos adquiridos, resultando em óbito com tempo de sobrevivência inferior a 60 horas. Nos potros do Grupo Debilitados, foi observada debilidade transitória imediata ao nascimento, sendo posteriormente demonstrada resposta satisfatória. No Grupo Saudáveis, foi observada melhor capacidade de adaptação neonatal, com ausência de alterações clínicas.

Os valores encontrados na avaliação da frequência cardíaca, respiratória e temperatura dos grupos estudados estão descritos na Tabela 1. Observaram-se bradicardia e hipotermia com $48 \mathrm{~h}$ de vida nos potros do Grupo Não Sobreviventes. Ainda no mesmo grupo, foi observada bradipneia na avaliação das 24h, com discreto aumento da frequência a partir de $36 \mathrm{~h}$ de vida 
Tabela 1. Descrição dos parâmetros clínicos de frequência cardíaca, respiratória e temperatura com médiaterro padrão da média, observados do nascimento até 48 horas de vida dos potros nos grupos estudados.

\begin{tabular}{|c|c|c|c|c|c|c|c|}
\hline & Grupos & Nascimento & 06 horas & 12 horas & 24 horas & 36 horas & 48 horas \\
\hline \multirow{3}{*}{$\begin{array}{l}\text { Frequência } \\
\text { cardíaca } \\
\text { (bpm) }\end{array}$} & $\begin{array}{c}\text { Não } \\
\text { sobreviventes }\end{array}$ & $77 \pm 2.5$ & $90 \pm 2$ & $84 \pm 4$ & $94 \pm 6$ & $98 \pm 14$ & 64 \\
\hline & Debilitados & $120 \pm 20$ & $110 \pm 10$ & $110 \pm 10$ & $120 \pm 20$ & $126 \pm 6$ & $120 \pm 20$ \\
\hline & Saudáveis & $86 \pm 14$ & $92 \pm 2$ & $94 \pm 2$ & $92 \pm 8$ & $74 \pm 14$ & $96 \pm 16$ \\
\hline \multirow{3}{*}{$\begin{array}{l}\text { Frequência } \\
\text { respiratória } \\
\quad(\mathrm{mpm})\end{array}$} & $\begin{array}{l}\text { Não } \\
\text { sobreviventes }\end{array}$ & $36 \pm 8$ & $48 \pm 16$ & $28 \pm 4$ & $16 \pm 4$ & $28 \pm 4$ & 36 \\
\hline & Debilitados & $44 \pm 4$ & $46 \pm 14$ & $34 \pm 14$ & $36 \pm 12$ & $36 \pm 12$ & $32 \pm 8$ \\
\hline & Saudáveis & $56 \pm 4$ & $42 \pm 14$ & $36 \pm 4$ & $48 \pm 8$ & $37 \pm 5$ & $50 \pm 14$ \\
\hline \multirow{3}{*}{$\begin{array}{c}\text { Temperatura } \\
\left({ }^{\circ} \mathrm{C}\right)\end{array}$} & $\begin{array}{c}\text { Não } \\
\text { sobreviventes }\end{array}$ & $37.5 \pm 1$ & $\begin{array}{c}38.1 \pm \\
0.3\end{array}$ & $37.8 \pm 0.7$ & $37.7 \pm 0.8$ & $36.9 \pm 1.5$ & 36.2 \\
\hline & Debilitados & $37.1 \pm 0.3$ & $36.5 \pm 0.2$ & $37.8 \pm 0.2$ & $38 \pm 0.1$ & $37.8 \pm 0.1$ & $38.6 \pm 0.1$ \\
\hline & Saudáveis & $37.7 \pm 0.4$ & $37.8 \pm 0.3$ & $37.7 \pm 0.1$ & $38.1 \pm 0.5$ & $38.4 \pm 0.1$ & $38.7 \pm 0.2$ \\
\hline
\end{tabular}

BPM (batimentos por minuto); MPM (movimentos por minuto)

Na avaliação dos potros do Grupo Debilitados, a hipotermia foi observada nas $6 \mathrm{~h}$, com estabilização da termorregulação nas $48 \mathrm{~h}$ de vida, alcançando valores semelhantes aos animais do Grupo Saudáveis.

Os parâmetros hematológicos dos potros neonatos estão descritos nas Tabelas 2 e 3. Em relação à hematimetria, foi observado que os neonatos comportaram-se de forma semelhante nos momentos avaliados. Observou-se que todos os potros apresentaram decréscimo dos parâmetros na avaliação imediata ao nascimento e nos demais momentos. Ao avaliar as Proteínas Plasmáticas Totais (PPT), foi observado incremento com $12 \mathrm{~h}$ nos potros do Grupo Saudáveis, caracterizando o momento pósmamada. Esse aumento não foi encontrado nos demais grupos. Em relação ao fibrinogênio, foi observado que os potros do Grupo Debilitados apresentaram maiores taxas imediatamente após o nascimento, mantendo níveis elevados até 48 horas de vida.

Tabela 2. Descrição de contagem de hemácias, hemoglobina, hematócrito e VCM com média土erro padrão da média, observados do nascimento até 48 horas de vida dos potros nos grupos estudados

\begin{tabular}{|c|c|c|c|c|c|}
\hline & Grupos & Nascimento & 12 horas & 24 horas & 48 horas \\
\hline \multirow{3}{*}{ Hemácias (x10 $)$} & $\begin{array}{c}\text { Não } \\
\text { sobreviventes }\end{array}$ & $9.9 \pm 0.2$ & $8.3 \pm 0.1$ & $7.3 \pm 0.1$ & 6.13 \\
\hline & Debilitados & $11.8 \pm 0.8$ & $9.4 \pm 1.2$ & $8.8 \pm 1.4$ & $8.5 \pm 1.2$ \\
\hline & Saudáveis & $9.5 \pm 0.5$ & $8.3 \pm 0.3$ & $9.1 \pm 0.7$ & $7.7 \pm 0.4$ \\
\hline \multirow{3}{*}{ Hemoglobina (g/dL) } & $\begin{array}{c}\text { Não } \\
\text { sobreviventes }\end{array}$ & $12.3 \pm 0.4$ & $10.3 \pm 0.5$ & $9 \pm 0.1$ & 7.2 \\
\hline & Debilitados & $13.7 \pm 0.6$ & $11.3 \pm 1.1$ & $10.4 \pm 1.4$ & $10 \pm 1.25$ \\
\hline & Saudáveis & $13 \pm 0.2$ & $11.1 \pm 0.5$ & $12.4 \pm 1.7$ & $10.3 \pm 0.3$ \\
\hline \multirow{3}{*}{ Hematócrito (\%) } & $\begin{array}{c}\text { Não } \\
\text { sobreviventes }\end{array}$ & $40.8 \pm 1.3$ & $34.2 \pm 0.7$ & $30.6 \pm 0.3$ & 23.4 \\
\hline & Debilitados & $46 \pm 1.3$ & $36.6 \pm 3.2$ & $33.4 \pm 4.8$ & $31.4 \pm 4.4$ \\
\hline & Saudáveis & $40.8 \pm 1.9$ & $35.2 \pm 1.4$ & $39 \pm 4.4$ & $33.6 \pm 0.3$ \\
\hline \multirow{3}{*}{ VCM (fl) } & $\begin{array}{c}\text { Não } \\
\text { sobreviventes }\end{array}$ & $41 \pm 0.4$ & $41.8 \pm 0.7$ & $41.6 \pm 0.1$ & 38.2 \\
\hline & Debilitados & $38.9 \pm 1.6$ & $38.5 \pm 1.5$ & $37.8 \pm 0.7$ & $36.8 \pm 0.2$ \\
\hline & Saudáveis & $42.6 \pm 0.5$ & $42.4 \pm 0.3$ & $42.3 \pm 1.2$ & $43.4 \pm 3.1$ \\
\hline
\end{tabular}


Tabela 3. Descrição de proteínas plasmáticas totais (PPT), fibrinogênio, plaquetas, leucócitos totais, neutrófilos, linfócitos e taxa neutrófilo:linfócito com média土erro padrão da média, observados do nascimento até 48 horas de vida dos potros nos grupos estudados

\begin{tabular}{|c|c|c|c|c|c|}
\hline & Grupos & Nascimento & 12 horas & 24 horas & 48 horas \\
\hline \multirow{3}{*}{ PPT (g/dL) } & $\begin{array}{c}\text { Não } \\
\text { sobreviventes }\end{array}$ & $5.3 \pm 0.1$ & $5.4 \pm 0.2$ & $5.9 \pm 0.3$ & 4.8 \\
\hline & Debilitados & $5.8 \pm 0.4$ & $5.9 \pm 0.1$ & $5.7 \pm 0.1$ & $5.9 \pm 0.1$ \\
\hline & Saudáveis & $4.8 \pm 0.5$ & $6.1 \pm 0.5$ & $6.3 \pm 0.6$ & $6.3 \pm 0.5$ \\
\hline \multirow{3}{*}{$\begin{array}{l}\text { Fibrinogênio } \\
\text { (mg/dL) }\end{array}$} & $\begin{array}{c}\text { Não } \\
\text { sobreviventes }\end{array}$ & $500 \pm 300$ & $400 \pm 200$ & $150 \pm 50$ & 200 \\
\hline & Debilitados & $900 \pm 100$ & $850 \pm 50$ & $650 \pm 50$ & $700 \pm 100$ \\
\hline & Saudáveis & $450 \pm 150$ & $550 \pm 250$ & $300 \pm 100$ & $600 \pm 100$ \\
\hline \multirow{3}{*}{ Plaquetas (x 10³) } & $\begin{array}{c}\text { Não } \\
\text { sobreviventes }\end{array}$ & $291 \pm 12.5$ & $230 \pm 40$ & $215 \pm 46$ & 218 \\
\hline & Debilitados & $402 \pm 81$ & $311 \pm 40$ & $363 \pm 121$ & $301 \pm 16$ \\
\hline & Saudáveis & $324 \pm 97$ & $243 \pm 98$ & $601 \pm 340$ & $387 \pm 96$ \\
\hline \multirow{3}{*}{$\begin{array}{l}\text { Leucócitos Totais } \\
\qquad(\mu L)\end{array}$} & $\begin{array}{c}\text { Não } \\
\text { sobreviventes }\end{array}$ & $4550 \pm 50$ & $6125 \pm 2475$ & $5050 \pm 2350$ & 800 \\
\hline & Debilitados & $6850 \pm 2150$ & $8750 \pm 1050$ & $8100 \pm 500$ & $12600 \pm 2600$ \\
\hline & Saudáveis & $4500 \pm 2500$ & $4475 \pm 1625$ & $5050 \pm 1350$ & $5700 \pm 1700$ \\
\hline \multirow{3}{*}{ Segmentados $(\mu L)$} & $\begin{array}{c}\text { Não } \\
\text { sobreviventes }\end{array}$ & $1413 \pm 243$ & $3850 \pm 2685$ & $2890 \pm 2215$ & $\mathrm{X}$ \\
\hline & Debilitados & $4842 \pm 1458$ & $7721 \pm 1099$ & $7027 \pm 111$ & $10736 \pm 2336$ \\
\hline & Saudáveis & $2965 \pm 2145$ & $2990 \pm 1341$ & $2455 \pm 23$ & $4115 \pm 1435$ \\
\hline \multirow{3}{*}{ Linfócitos $(\mu \mathrm{L})$} & $\begin{array}{c}\text { Não } \\
\text { sobreviventes }\end{array}$ & $3045 \pm 239$ & $2147 \pm 255$ & $2072 \pm 74$ & $\mathrm{X}$ \\
\hline & Debilitados & $1892 \pm 717$ & $826 \pm 56$ & $987 \pm 303$ & $1460 \pm 60$ \\
\hline & Saudáveis & $1435 \pm 315$ & $1460 \pm 308$ & $2384 \pm 1200$ & $1246 \pm 86$ \\
\hline \multirow{3}{*}{ Taxa N:L } & $\begin{array}{c}\text { Não } \\
\text { sobreviventes }\end{array}$ & $1: 3$ & $3: 2$ & $2: 2$ & $X$ \\
\hline & Debilitados & $4: 1$ & $7: 1$ & $7: 1$ & $10: 1$ \\
\hline & Saudáveis & $2: 1$ & $2: 1$ & $2: 2$ & $4: 1$ \\
\hline
\end{tabular}

\section{DISCUSSÃo}

O grupo dos potros Saudáveis apresentou maior tempo de gestação ( $320 \pm 2$ dias) e maior intervalo inoculação-parto (20,5 $\pm 2,5$ dias). Segundo Koterba (1990a), o nascimento do potro antes dos 320 dias de gestação é considerado prematuro. A dismaturidade é atribuída aos animais que nascem dentro do período gestacional fisiológico ( $\geq 320$ dias), porém apresentam características de imaturidade, como baixo peso ao nascer (Koterba, 1990a). Assim, os neonatos do grupo Não Sobreviventes e Debilitados podem ser classificados como prematuros, sendo os potros Saudáveis considerados dismaturos.

No presente estudo, observou-se que a resposta frente ao tratamento é variável; fato demonstrado pelos distintos resultados gestacionais $\mathrm{e}$ neonatais. Essa diferença de resposta também é encontrada em estudos semelhantes, como o realizado por Bailey et al. (2010), sobre indução de placentite ascendente com infusão intracervical de S. equi zooepidemicus, no qual se demonstrou que o tratamento prolongou a gestação por mais tempo nas éguas tratadas, porém a sobrevivência neonatal não foi diferente em comparação ao grupo não tratado. Para Bain (2004), os fatores determinantes mais importantes para o resultado neonatal estão relacionados com o desenvolvimento fetal e o tempo de injúria placentária.

Sugere-se que a maturação fetal precoce não é necessariamente acompanhada por maturação completa de todos os sistemas orgânicos, visto que, nos potros prematuros, observou-se deficit na adaptação extrauterina. Apesar da dismaturidade dos potros Saudáveis, estes 
apresentaram melhor função adaptativa e capacidade de resposta em comparação aos demais neonatos; o que pode ser atribuído ao maior tempo de gestação após a indução de placentite, já que o Grupo Não Sobreviventes obteve média de $12 \pm 2$ dias e resultou no nascimento de potros prematuros e severamente debilitados. Segundo Rossdale et al. (1991) e Mays et al. (2002), pode ocorrer maturação fetal acelerada em éguas que mantêm a gestação por período maior, caracterizando o estágio crônico da placentite. Embora a maturação fetal ocorra de forma precoce e incompleta, pode promover o nascimento de um potro viável com maior probabilidade de sobrevivência.

Na avaliação dos reflexos posturais de potros saudáveis, são descritos na literatura os seguintes tempos após o nascimento: decúbito esternal, 510 minutos; reflexo de sucção, 5-20 minutos; levantar-se e permanecer em estação, até uma hora (Koterba, 1990b; Stoneham, 2006).

No presente estudo, foi observado que somente os potros saudáveis apresentaram-se dentro dos limites fisiológicos para permanência em decúbito esternal. Contudo, na apresentação do reflexo de sucção, demoraram mais tempo que o aceitável para neonatos hígidos, o que evidencia leve debilidade devido ao atraso. Os demais neonatos deste estudo apresentaram claramente deficit nessa avaliação, demonstrando disfunção na adaptação extrauterina. Segundo Irvine (1984), o atraso do reflexo de sucção indica imaturidade neuromotora, resultando em incoordenação. Em relação ao tempo para se levantar, todos os neonatos estavam dentro dos limites descritos para potros saudáveis (à exceção do quarto neonato).

Na avaliação da atividade respiratória, os potros debilitados e saudáveis apresentaram frequência dentro dos limites fisiológicos (40-60mpm após o nascimento; 30-40mpm nas primeiras horas de vida), conforme descrito por Stoneham (2006). No entanto, os potros não sobreviventes apresentaram momentos marcantes de bradipneia. Segundo Koterba (1990b), essa hipoventilação é comum em potros prematuros e que sofreram hipóxia. Segundo Orsini (2011), a variação nestes parâmetros (acima ou abaixo dos limites fisiológicos) está negativamente relacionada com sobrevivência.
Em todos os momentos de avaliação, os neonatos do grupo Debilitados e do Saudáveis apresentaram frequência cardíaca dentro dos limites fisiológicos (80-120 bpm), conforme descrito por Stoneham (2006). No grupo Não Sobreviventes, a redução da atividade cardíaca (64 bpm) ocorreu juntamente com a diminuição na temperatura corporal $\left(36.2^{\circ}\right)$, com $48 \mathrm{~h}$ de vida. Segundo Corley (2003), a redução da frequência cardíaca ocorre em potros com hipotermia.

A temperatura corporal em potros neonatos varia de $37-39^{\circ} \mathrm{C}$ do nascimento até os primeiros dias de vida, conforme descrito por Stoneham (2006). A hipotermia $\left(36,2^{\circ} \mathrm{C}\right)$ observada nos neonatos não sobreviventes nas $48 \mathrm{~h}$ demonstra a imaturidade orgânica desses indivíduos, já que os mecanismos termogênicos estão relacionados com a maturação do eixo hipotalâmicohipofisiário-adrenal, segundo Silver et al. (1991). Ainda, para Ousey et al. (1997), o deficit de isolamento favorece a perda de calor em potros imaturos, como baixo peso ao nascer e pelagem curta e fina, conforme foi observado nos neonatos do presente estudo. A falha na termorregulação observada nos potros não sobreviventes sugere imaturidade neuroendócrina, sendo a perda de calor maior que a taxa de produção.

$\mathrm{Na}$ avaliação hematológica, com exceção do volume corpuscular médio (VCM), todos os potros apresentaram redução nos valores do eritrograma. Segundo Jeffcott et al. (1982), ocorre progressiva redução do tamanho dos eritrócitos durante a segunda metade da gestação. Devido a isso, potros prematuros podem apresentar baixa contagem de hemácias, menor concentração de hemoglobina e baixo hematócrito, além de alto VCM, quando comparados com potros a termo. No presente estudo, foi observada redução dos valores após o nascimento; no entanto, sem alteração do VCM. Segundo Axon e Palmer (2008), imediatamente após o nascimento a concentração de hemoglobina e hematócrito aumentam transitoriamente e decrescem, em média, 10\% nas $12-24$ h seguintes.

Foi observado incremento das PPT após o nascimento somente nos potros considerados saudáveis. Segundo Lester (2005), potros prematuros podem ser incapazes de tolerar a 
mesma quantidade de alimento e nutrientes que potros hígidos. Com isso, pode-se sugerir que o incremento proteico refletiu o grau de maturação e a capacidade de absorção intestinal nos neonatos saudáveis. No entanto, a manutenção dos baixos níveis de PPT, do nascimento até 48h, nos potros não sobreviventes e debilitados, permite sugerir a falha na absorção proteica e/ou intenso catabolismo.

Na avaliação da concentração de fibrinogênio, nota-se que todos os neonatos apresentaram taxas elevadas ao nascimento. Esses dados corroboram a afirmação de Morresey (2005), de que a hiperfibrinogenemia é considerada bom indicador prognóstico em casos de potros prematuros que foram expostos a agentes infecciosos pré-parto, sendo interpretado como resposta neonatal satisfatória.

No leucograma, foi evidenciado que os potros não sobreviventes e os saudáveis apresentaram baixa contagem de leucócitos totais no nascimento, demonstrando discretas variações até as 48h. Segundo Morresey (2005), é característico observar leucopenia em potros imaturos, assim como a taxa neutrófilo:linfócito $(\mathrm{N}: \mathrm{L})$ em torno de 1 é fortemente sugestiva de despreparo para o nascimento.

Segundo Rossdale (2004), três horas após o nascimento ocorre neutrofilia em resposta ao aumento do cortisol plasmático. O incremento também ocorre na proporção $\mathrm{N}: \mathrm{L}$, a qual, no nascimento, é em torno de 2.7:1, podendo atingir concentração de 4:1. O prognóstico desfavorável é atribuído a potros prematuros que apresentam neutropenia e taxa $\mathrm{N}: \mathrm{L}$ menor que 1.5 durante as primeiras $24 \mathrm{~h}$ de vida. No presente estudo, somente os potros debilitados apresentaram neutrofilia nas $12 \mathrm{~h}$, com manutenção dos valores até 48h. Não foi observada neutrofilia e mudança consistente na taxa $\mathrm{N}: \mathrm{L}$ nas primeiras $24 \mathrm{~h}$ de vida dos potros não sobreviventes e dos saudáveis. Isso reafirma a imaturidade orgânica desses indivíduos.

\section{CONCLUSÃO}

Os potros nascidos de éguas com placentite ascendente e tratadas demonstraram evolução clínica e respostas neonatais distintas. Embora com discreto atraso no reflexo de sucção, somente os potros dismaturos apresentaram reflexos posturais e comportamentais próximos do adequado. Quanto maior o tempo de manutenção da gestação após a injúria placentária, melhor será a maturação fetal, o que refletirá em viabilidade e melhor capacidade de resposta neonatal.

\section{AGRADECIMENTOS}

Ouro Fino Agronegócio, FAPERGS, CNPQ, CAPES.

\section{REFERÊNCIAS}

AXON, J.E.; PALMER, J.E. Clinical Patology of the foal. Vet. Equine Clin., v.24, p.357-385, 2008.

BAILEY, C.S; MACPHERSON, M.L.; POZOR, M.A. et al. Treatment efficacy of trimethoprim sulphametoxazole, pentoxifilyne and altrenogest in experimentally induced equine placentitis. Theriogenology, v.74, p.402-412, 2010.

BAIN, F.T. Management of the foal from the mare with placentitis: A clinician's approach. In: Proceedings. Annual convention of the American Association of Equine Practitioners, 50., 2004, Denver, EUA, p.1419-1204.

CORLEY, K.T.T. Monitoring and treating the cardiovascular system in neonatal foals. Clin. Tech. Equine Pract., v.2, p.42-55, 2003.

GILES, R.C.; DONAHUE, J.M.; HONG, C.B. et al. Causes of abortion, stillbirth, and perinatal death in horses - 3,527 cases (1986 -1991). J. Am. Vet. Med. Assoc., v.203, p.1170-1175, 1993.

HONG, C.B.; DONAHUE, J.M.; GILES, R.C. et al. Etiology and pathology of equine placentitis. J. Vet. Diagn. Invest., v.5, p.56-63,1993.

IRVINE, C.H.G. Hypothyroidism in the foal. Equine Vet. J., v.16, p.302-306, 1984.

JEFFCOTT, L.B.; ROSSDALE, P.D.; LEADON, D.P. Haematological changes in the neonatal period of normal and induced premature foals. J. Reprod. Fertil., v.32, Suppl., p.537-544, 1982.

KOTERBA, A.M. Prematurity. In: KOTERBA, A.M.; DRUMMOND. W.H.; KOSCH, PC (Ed.) Equine Clinical Neonatology. Philadelphia, Lea \& Febiger, 1990a. p.54-70. 
KOTERBA, A.M. Physical examination. In: KOTERBA, A.M.; DRUMMOND, W.H.; $\mathrm{KOSCH}$, P.C. (Ed.). Equine Clinical Neonatology. Philadelphia: Lea \& Febiger, 1990b. p.71-85.

LESTER, G. Maturity of the neonatal foal. Vet. Clin. Equine, v.21, p.333-355, 2005.

MAYS, M.B.C.; LE BLANC, M.M.; PACCAMONTI, D. Route of fetal infection in a model of ascending placentitis. Theriogenology, v.58, p.791-792, 2002.

MORRESEY, P.R. Prenatal and perinatal indicators of neonatal viability. Clin. Tec. Equine Prac., v.4, p.238-249, 2005.

ORSINI, J.A. A fresh look at the process of arriving at a clinical prognosis. Part 3: Neonatal illness. J. Equine Vet. Sci., v.31, p.434-446, 2011.

OUSEY, J.C.; MCARTHUR, J.A.; ROSSDALE, P.D. Thermoregulation in sick foals aged less than one week. The Vet. J., v.153, p.185-196, 1997.

ROSSDALE, P.D.; SILVER, M. The concept of readiness for birth. J. Reprod. Fertil. Suppl., v.32, p.507-510, 1982.
ROSSDALE, P.D.; OUSEY, J.C.; COTTRILL, C.M. et al. Effects of placental pathology on maternal plasma progestagen and mammary secretion calcium concentrations and on neonatal adrenocortical function in the horse. J. Reprod. Fertil. Suppl., v.44, p.579-590, 1991.

ROSSDALE, P.D.; OUSEY, J.C.; CHAVATTE, P. Readiness for birth: an endocrinological duet between fetal foal and mare. Equine Vet. J., v.24, p.96-99, 1997.

ROSSDALE, P.D. The maladjusted foal: Influences of intrauterine growth retardation and birth trauma. In: Proceedings. Annual convention of the American Association of Equine Practitioners, 50., 2004, Denver, EUA, p.75-126.

SILVER, M.; FOWDEN, A.L.; KNOX, I. et al. Relationship between circulating hiiodothyronine and cottisol in the perinatal period in the foal. J. Reprod. Fertil. Suppl., v.44, p.619626, 1991.

STONEHAM, S.J. Assessing the newborn foal. In: PARADIS, M.R. (Ed.). Equine Neonatal Medicine. Philadelphia: Elsevier Saunders, 2006. p.31-38. 